\title{
Alteration of tumor markers may predict survival in colorectal cancer patients treated with TAS-102 or regorafenib as salvage-line chemotherapy: a single- institutional experience
}

Keiji Matsuda $^{1 *}$, Keijiro Nozawa ${ }^{1}$, Kohei Ohno ${ }^{1}$, Yuka Okada ${ }^{1}$, Takahiro Yagi ${ }^{1}$, Mitsuo Tsukamoto ${ }^{1}$, Yoshihisa Fukushima ${ }^{1}$, Takuya Akahane ${ }^{1}$, Atsushi Horiuchi ${ }^{1}$, Ryu Shimada ${ }^{1}$, Tamuro Hayama ${ }^{1}$, Koichi Okamoto ${ }^{1}$, Takeshi Tsuchiya ${ }^{1}$, Junko Tamura ${ }^{1}$, Hisae Iinuma ${ }^{1}$, Yuko Sasajima ${ }^{2}$, Fukuo Kondo ${ }^{2}$, Shoichi Fujii ${ }^{3}$ and Yojiro Hashiguchi ${ }^{1}$

${ }^{1}$ The Department of Surgery, Teikyo University School of Medicine, Japan

${ }^{2}$ The Department of Pathology, Teikyo University School of Medicine, Japan

${ }^{3}$ The Department of Surgery, Kagaku Ryoho Kenkyujo Byoin, Japan

\begin{abstract}
Objectives: TAS-102 and regorafenib are novel antineoplastic drugs recommended for salvage-line chemotherapy. The objective of this study was to elucidate useful markers with predictive values for the effectiveness of these drugs. Methods: Between August 2013 and April 2016, 23 patients with refractory colorectal cancer received salvage-line chemotherapy at Teikyo University Hospital, Japan. 15 patients received TAS-102 monotherapy and 15 received regorafenib, including seven who had dual therapies. Tumor markers were analyzed for possible correlations with tumor response and the patients' prognoses after these treatments. Results: Twelve patients of each group had radiologically measurable tumors. None of the TAS-102-treated patients achieved complete response (CR) or partial response (PR). After regorafenib therapy, no patients achieved CR, but one (8\%) patient showed PR. These and the lack of correlation between the tumor responses and the patients' overall survival (OS) suggested a limited predictive value of RECIST-based tumor evaluation in our study. Nonetheless, the OS of the patients with a decreased CA19-9 level after initial treatment with TAS-102 tended to be longer than that of the patients with an increased CA19-9 level ( $\mathrm{p}=0.058)$. The OS of the patients with decreased CEA after initial regorafenib treatment was significantly longer than that of patients with increased CEA ( $p=0.03$ ). Conclusions: The results of the present analysis suggest that CEA and CA19-9 may be more practical and useful as predictive/prognostic markers for refractory CRC patients treated with TAS-102 and regorafenib even when the predictive value of the tumor response measured using RECIST is not clear.
\end{abstract}

\section{Introduction}

Colorectal cancers (CRCs) are the second leading cause of cancer death in Japan, and the number of patients dying of CRC is steadily increasing [1,2]. With advances in systemic therapy for metastatic CRC, the survival of these patients has been prolonged by up to 20 months with fluorouracil (FU), irinotecan, oxaliplatin and molecular targeted agents [3]. However, most patients develop resistance to these drugs and experience disease progression. Despite the diverse currently acceptable cytotoxic chemotherapy and/or biologic agents that enable a considerable portion of CRC patients to survive and show good performance status, the refractory patients have relatively few additional treatment options.

TAS-102 is an orally administered combination of a thymidinebased nucleic acid analogue, trifluridine, and a thymidine phosphorylase inhibitor, tipiracil hydrochloride [4-7]. Trifluridine is the active cytotoxic component of TAS-102, and tipiracil hydrochloride is a potent inhibitor of thymidine phosphorylase. Regorafenib is a novel oral multikinase inhibitor that blocks the activity of several protein kinases, including kinases involved in the regulation of tumor angiogenesis, oncogenesis and the tumor microenvironment [8]. A Phase 3 trial showed that TAS- 102 and regorafenib were associated with a significant improvement in overall survival [8-11]. However, to our knowledge, biomarkers have not been found to refine the population of CRC patients likely to obtain benefit from TAS-102 and regorafenib.

We conducted the present study to retrospectively evaluate the clinical benefit and tolerability of TAS-102 and regorafenib monotherapy in patients with metastatic CRC that failed standard treatments. We further analyzed the alterations of the levels in serum of carcinoembryonic antigen (CEA) and carbohydrate antigen 19-9 (CA19-9) during treatments with TAS-102 and regorafenib in order to examine whether CEA and CA19-9 are useful as predictive and prognostic markers for refractory colorectal cancer patients.

\section{Methods}

\section{Study population}

Between August 2013 and April 2016, 15 Japanese patients with refractory CRC received TAS-102 monotherapy and 15 patients

Correspondence to: Keiji Matsuda, M.D., Ph.D., The Department of Surgery, Teikyo University School of Medicine, 2-11-1 Kaga, Itabashi-ku, Tokyo, Japan, Tel: 81-3-3964-1211; Fax: 81-3-5375-6097; E-mail: keiji@med.teikyo-u.ac.jp

Key words: TAS-102, regorafenib, prognostic marker, CEA, CA19-9

Received: March 10, 2017; Accepted: April 12, 2017; Published: April 15, 2017 
Matsuda K (2017) Alteration of tumor markers may predict survival in colorectal cancer patients treated with TAS-102 or regorafenib as salvage-line chemotherapy: a single-institutional experience

received regorafenib as salvage treatment at the surgical department of Teikyo University Hospital. All patients had previously received $5 \mathrm{FU}$, irinotecan, or oxaliplatin with or without biologic agents such as cetuximab, panitumumab and bevacizumab. The case inclusion criteria were (1) age >18 years; (2) pathologically or clinically proven metastatic CRC; (3) adequate bone marrow, liver, and renal function. All participants provided written informed consent before starting TAS-102 or regorafenib. The study was retrospectively performed in accord with the Declaration of Helsinki and was approved by the Hospital's institutional review board.

\section{Chemotherapy}

TAS- 102 at $35 \mathrm{mg} / \mathrm{m}^{2}$ was administered twice daily, 5 days/week, with 2 days of rest as an interval, over a 2 -weeks period. This was, followed by a 14-day rest period, thus completing one treatment cycle [10]. The regimen was repeated every 4 weeks. Patients were followed up every week during the first two cycles and every 2 weeks from cycle 3. Prespecified dose reductions and a delay of the following cycle were allowed for the management of adverse events.

As regorafenib treatment, patients received oral regorafenib 160 mg once daily for the first 3 weeks of each 4 -week cycle. Patients were followed up every week during the first two cycles and then every 2 weeks from cycle 3. Prespecified dose reduction (to 120 or $80 \mathrm{mg}$ ) and a delay of the following cycle (up to 28 days) were allowed for the management of adverse events.

\section{Assessments}

Blood samples were taken once per month to measure the levels of the tumor markers, CEA and CA19-9, in serum. The tumor response was assessed with a computed tomography scan every 2-3 months. The tumor response was evaluated using a method identical to that used in the Response Evaluation Criteria in Solid Tumor (RECIST) ver. 1.1 [12]. Toxicities were assessed according to the U.S. National Cancer Institute's Common Toxicity Criteria for Adverse Events (CTCAE) ver. 3.0. In the present study we defined the subgroup of 'within normal limits' for CEA using the $0-4.9 \mathrm{ng} / \mathrm{ml}$ as the normal limits and, for the remaining patients, we subsequently defined the 'CEA increase subgroup' as the patients whose CEA values increased continuously during their TAS-102 or regorafenib therapy, and the 'CEA decrease subgroup' as the patients whose CEA values decreased from the previous value on at least one occasion during TAS-102 or regorafenib therapy. Likewise, the normal limits 0-37 U/ml was used to define the 'CA19-9 within normal limits subgroup', and subsequently 'CA19-9 increase subgroup' and 'CA19-9 decrease subgroup' were similarly defined.

As for KRAS, cancer tissue was collected from thin slices manufactured from the paraffin block at the pathological department of Teikyo University Hospital. The cancer tissue was sent to an inspection facility, and KRAS status was examined focusing on the codon 12/13, by PCR-rSSO method (Kagakuhoken Kenkyujo Company, Yokohama, Japan).

\section{Statistical analysis}

Descriptive statistics were calculated as proportions and medians. Treatment outcomes were estimated as the response rate, overall survival (OS), and toxicities. The response rate was determined according to RECIST ver. 1.1 [12] based on the tumor size measured before the treatment protocol and that measured using the first radiological image obtained after starting the treatment protocol. Of note, RECIST utilizes the sum of one-dimensional measurements of the greatest diameter of the tumor and/or metastasis. complete response (CR) is defined as complete absence of disease, partial response (PR) as $>30 \%$ decrease of the sum of the greatest diameters, progressive disease (PD) as an increase of $>20 \%$ of the sum of the greatest diameters and stable disease (SD) as all outcomes in between. The OS was defined as the time from the first TAS-102 or regorafenib treatment to death from any cause.

The survival data were assessed using the Kaplan-Meier method. All analyses were performed using Stat Flex ver. 6.0 software (Artech, Osaka, Japan).

\section{Results}

\section{Patients}

The patients' baseline characteristics are summarized in Table 1. The characteristics of the 15 patients who received TAS-102 and the 15 who received regorafenib were essentially identical. Regorafenib was administered to five patients before TAS-102 administration, and TAS-102 was administered to two patients before regorafenib administration.

\section{Toxicity}

Toxicities of TAS-102 of $\geq$ grade 3 included leukopenia (20\%), neutropenia (20\%), anemia (13\%), hearing difficulty $(9 \%)$ and decreased appetite (9\%). There was no treatment-related death. Adverse events following regorafenib treatment included hand-foot syndrome (13\%), hypertension(13\%), decreased appetite(7\%), hypertransaminase (7\%), stomatitis (7\%), hyperglycemia (7\%), low sodium (7\%), high potassium (7\%), and thrombopenia (7\%). The incidence of adverse events thus differed between the TAS-102 and regorafenib groups, although the small sample size prevented us from statistical tests, calling for future studies on greater scales.

\section{Alteration of tumor markers}

The alterations of serum levels of the tumor markers during the TAS-102 and regorafenib therapies are summarized in Table 2. Among the TAS-102-treated patients, eight (53\%) belonged to the CEA increase group, and five (33\%) patients belonged to the CEA decrease group; seven (47\%) cases belonged to the CA19-9 increase group and 5 (33\%) patients to the CA19-9 decrease group. Among the regorafenibtreated patients, $11(73 \%)$ were in the CEA decrease group and seven (47\%) were in the CA19-9 decrease group.

\section{Chemotherapy discontinuation}

The average period of TAS-102 administration was 3.5 months, and that of regorafenib was 3.8 months. Treatment was discontinued in eight (53\%) TAS-102 patients, and 13 (87\%) regorafenib patients. The causes of discontinuation of TAS-102 were disease progression in four cases, intolerable adverse events in two cases, deterioration of general condition in one case, and patient's wish in one case. The reasons for regorafenib discontinuation were mostly similar to those for TAS-102 (details not shown).

The tumor responses assessed using the RECIST criteria are listed in Table 3. TAS-102 treatment resulted in no complete response (CR), no partial response (PR). Stable disease (SD) in five patients and progressive disease (PD) in seven patients. Regorafenib monotherapy resulted in no $\mathrm{CR}$, one $\mathrm{PR}$, three $\mathrm{SD}$ and eight $\mathrm{PD}$ patients.

Predictive factors for OS after TAS-102 and regorafenib treatments were considered as summarized in Table 4 . The OS was compared 
Matsuda K (2017) Alteration of tumor markers may predict survival in colorectal cancer patients treated with TAS-102 or regorafenib as salvage-line chemotherapy: a single-institutional experience

Table 1. Baseline patient characteristics

\begin{tabular}{|c|c|c|}
\hline & TAS-102 & regorafenib \\
\hline Characteristics & No.(\%) & No.(\%) \\
\hline Gender & & $11(73)$ \\
\hline male & $11(73)$ & $4(27)$ \\
\hline female & $4(27)$ & $7(47)$ \\
\hline Primary site of disease & & $8(53)$ \\
\hline colon & $10(67)$ & \\
\hline rectum & $5(33)$ & $9(60)$ \\
\hline Tumor KRAS mutation status & & $6(40)$ \\
\hline wild-type & $8(53)$ & \\
\hline mutated & $7(47)$ & $9(60)$ \\
\hline $\begin{array}{c}\text { Average No. of previous } \\
\text { systemic anticancer } \\
\text { therapies }\end{array}$ & & $6(40)$ \\
\hline $01-M a r$ & $8(53)$ & \\
\hline$\geqq 4$ & $7(47)$ & $14(93)$ \\
\hline Previous treatment & & $9(60)$ \\
\hline anti-VEGF & $14(93)$ & $2(13)$ \\
\hline anti-EGFR & $8(53)$ & $0(0)$ \\
\hline TAS-102 & $0(0)$ & \\
\hline regorafenib & $5(33)$ & \\
\hline & & \\
\hline & & \\
\hline & & \\
\hline & & \\
\hline & & \\
\hline & & \\
\hline & & \\
\hline & & \\
\hline & & \\
\hline
\end{tabular}

Table 2. Change of tumor markers

\begin{tabular}{|c|c|c|}
\hline & TAS-102 & regorafenib \\
\hline & No.(\%) & No.(\%) \\
\hline & CEA & $4(27)$ \\
\hline increase & $8(53)$ & $11(73)$ \\
\hline decrease & $5(33)$ & $0(0)$ \\
\hline within normal limits & $2(13)$ & \\
\hline & CA19-9 & $4(27)$ \\
\hline increase & $7(47)$ & $7(47)$ \\
\hline decrease & $5(33)$ & $4(27)$ \\
\hline within normal limits & $3(20)$ & \\
\hline
\end{tabular}

Table 3. Tumor Response by RECIST criteria

\begin{tabular}{|c|c|c|}
\hline & TAS-102 & regorafenib \\
\hline Treatment outcome & No. & No. \\
\hline Response & & 0 \\
\hline Complete response (CR) & 0 & $1(8 \%)^{*}$ \\
\hline Partial response (PR) & 0 & $3(25 \%)^{*}$ \\
\hline Stable disease (SD) & $5(42 \%)^{*}$ & $8(67 \%)^{*}$ \\
\hline Progressive disease (PD) & $7(58 \%)^{*}$ & $3(20 \%)$ \\
\hline Not available & $3(20 \%)$ & 8.3 \\
\hline Response rate(\%) & 0 & $33^{*}$ \\
\hline Disease control rate(\%) & $42^{*}$ & \\
\hline
\end{tabular}

*Percentage was calculated excluding cases which are not available

between subgroups, i.e., sex, age, location, tumor KRAS status, past administration, alteration of CEA, alteration of CA19-9 and RECIST evaluation (Table 4, Figures1 and 2). The OS of the patients in the CA19-9 decrease subgroup tended to be longer than that of the patients in the CA19-9 increased subgroup when TAS-102 treatment was used (Figure1b). The OS of the patients in the CEA decreased subgroup was significantly longer than that of the patients in the CEA increased subgroup when regorafenib therapy was used (Figure 2a). Thus, these findings point to a view that CA19-9 and CEA have predictive values for TAS-treated patients and regorafenib-treated patients, respectively. However, when subgroups were obtained based on the RECIST evaluation, no significant difference in OS was observed between the patients in the SD group and those in the PD group when TAS-102 was given (Figure $3 \mathrm{a}$ ).
In the patients given regorafenib, the OS of the patients in the PR and SD groups tended to be better than that of the patients in the PD group (Figure 3b). No significant correlations were confirmed between the RECIST evaluations and the alterations of tumor markers by the selection of anticancer drug (details not shown).

\section{Discussion}

TAS-102 and regorafenib were recommended for salvage-line chemotherapy in both the 2016 Japanese Society for Cancer of the Colon and Rectum (JSCCR) Guidelines for the treatment of colorectal cancer [13] and the U.S. NCCI guidelines [14].

Regarding TAS-102 toxicities, our present findings are almost identical to the data obtained in the RECOURSE trial [10]. Regarding regorafenib, the frequency of hand foot syndrome of $\geq$ grade 3 was $13 \%$, in accordance with the result of the CORRECT trial [8].

The impact of chemotherapy on metastatic colorectal cancer reportedly decreases with the transition of sequential chemotherapy. First-line chemotherapy potentially has the highest expected response rate [15], and much less impact is expected from salvageline chemotherapy $[8,10]$. For example, the response rate to first-line colorectal cancer chemotherapy has been $58 \%-62 \%$ [15], but $1.0 \%-$

TAS-102
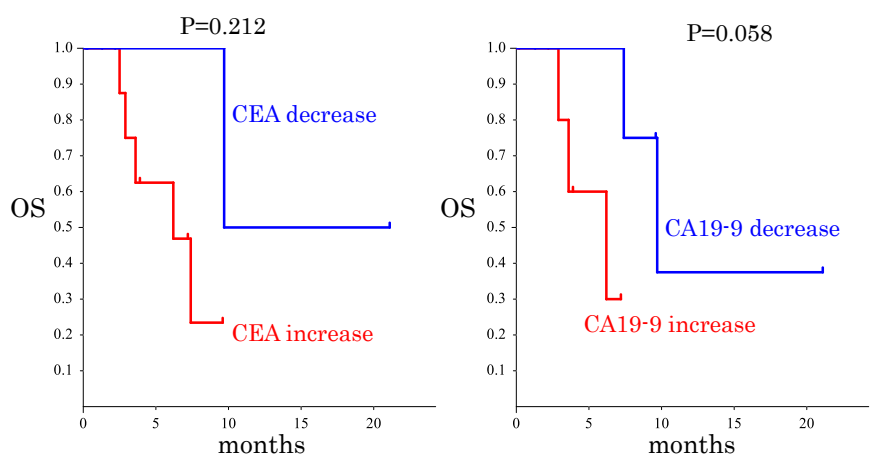

Figure 1. Kaplan-Meier plots for overall survival (OS) time for the patients treated with TAS-102. (a) Comparison of OS between the CEA decrease and the CEA increase subgroups of the patients who received the TAS therapy. The subgroups were defined based on the level and change in CEA values during TAS-102 treatment as described in the 'assessments' section. (b) Similar to (a) but the CA19-9 decrease subgroup was compared with the CA19-9 increase subgroup.

$$
\text { regorafenib }
$$
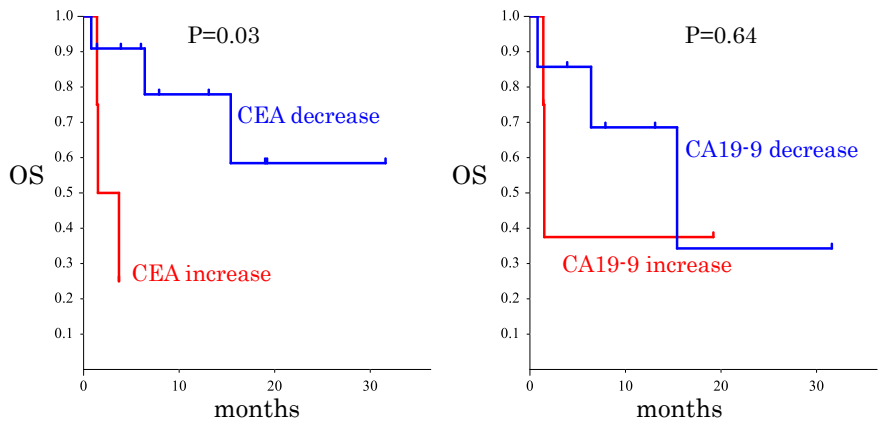

Figure 2. Kaplan-Meier plots for overall survival (OS) time for the patients treated with regorafenib analyzed similarly to Figure 1. (a) Comparison of OS between the CEA decrease and the CEA increase subgroups. (b) Similar to (a) but the the CA19-9 decrease subgroup was compared with the CA19-9 increase one. 
TAS-102

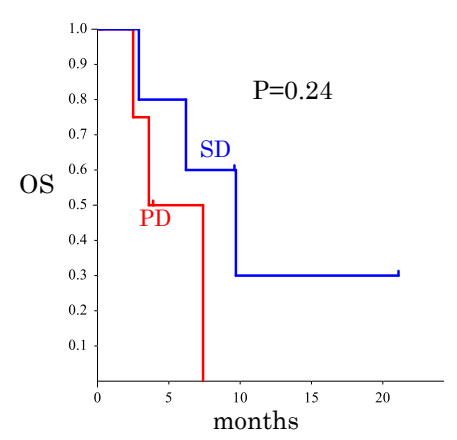

regorafenib

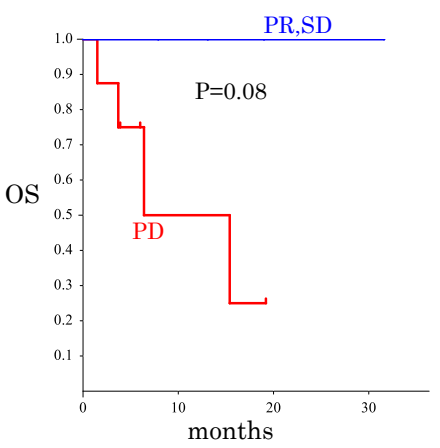

Figure 3. Kaplan-Meier plots for overall survival (OS) time for the patients treated with TAS-102. (a) Comparison between the stable disease (SD) subgroup and the progressive disease (PD) subgroup defined by RECIST criteria applied before and after the initial TAS102 treatment. (b) Similar to (a) but the PD subgroup and the subgroup that merged the partial response (PR) and SD subgroups based on the RECIST criteria applied before and after the initial regorafenib were compared

Table 4. Univariate analysis for prognostic factors for overall survival after TAS-102 and regorafenib treatments

\begin{tabular}{|c|c|c|}
\hline & TAS-102 & regorafenib \\
\hline & P-value & P-value \\
\hline Male vs. female & 0.92 & 0.49 \\
\hline Age $(<65$ vs 65 $\leqq$ ) & 0.57 & 0.81 \\
\hline Location 1:colon vs. rectum & 0.54 & $\underline{\mathbf{0 . 0 2}}$ \\
\hline Location 2:right-sided vs. left-sided & 0.92 & $\mathbf{0 . 0 6}$ \\
\hline KRAS wild-type vs. mutant & 0.83 & 0.83 \\
\hline Past administration:anti-VEGF vs. anti-VEGF\&EGFR & 0.79 & 0.56 \\
\hline CEA decrease vs. CEA increase & 0.21 & $\mathbf{0 . 0 3}$ \\
\hline CA19-9 decrease vs. CA19-9 increase & $\mathbf{0 . 0 5 8}$ & 0.64 \\
\hline PR/SD vs. PD & 0.24 & 0.08 \\
\hline
\end{tabular}

$1.6 \%$ for salvage-line therapies. They may underlie the difficulty in assessing the effects of such drugs, according to the RECIST trial.

The reported median durations of treatment with salvage chemotherapy were 6.8 weeks for regorafenib [8] and 6.7 weeks for TAS-102 [10], which are much shorter than those of the first- and second-line chemotherapies in the same patients. Some patients treated with salvage-line chemotherapy are too exhausted to undergo a followup imaging study, or they die of cancer without undergoing a follow-up examination. Therefore, it may not be possible to sufficiently perform imaging studies to evaluate the effects of these salvage chemotherapies. In addition, the evaluation of tumors with no measurable lesion, (such as disseminated lesions,) according to the RECIST criteria would not be feasible. It is also difficult to distinguish between necrotic tissue and the viable tumor [16].

In contrast, the measurement of CEA and CA19-9 is easy, objective and informative even for exhausted patients treated with salvage-line chemotherapy. Huang et al. reported that a CEA ratio defined as postchemotherapy-CEA/pre-CEA was a good prognostic factor in Stage IV CRC patients treated with chemotherapy, and that this ratio was correlated with the RECIST evaluation after first-line chemotherapy [16]. However, there have been no reports that address such particular benefits of tumor markers to evaluate the effects of salvage-line chemotherapy or to predict the patients' prognoses. The results of the present study demonstrated that these tumor markers could be used as prognostic factors even for salvage-line chemotherapy.

Regarding TAS-102 and regorafenib, few markers have been reported to be able to predict long survival from short survival.
Tabernero et al. reported that regorafenib was associated with a clinical benefit in patient subgroups based on PIK3CA wild-type and KRAS wild-type [17]. It is necessary to identify more predictive parameters to select patients who are likely to benefit from TAS-102 or regorafenib. Our present findings indicate that well-known tumor markers might be more useful and practical than the RECIST criteria in the decisionmaking regarding salvage chemotherapy for CRC patients. Our analysis revealed predictive values distinct between CEA and CA19-9 depending on the anticancer drug, but we suggest that both CEA and CA19-9 should be measured and studied in clinical practice.

The limitations of the present study are that the study was retrospective and that our data were derived from a small number of patients. Our findings should be confirmed in a prospective clinical trial on a greater scale.

In conclusion, the results of our analysis suggest that the measurement of serum CEA and CA-19-9 may be more practical and useful prognostic markers for refractory colorectal cancer patients treated with TAS-102 and regorafenib compared to a RECIST evaluation.

\section{Conflicts of interest}

The authors declare that there is no conflict of interest.

\section{References}

1. National Cancer Institute, http://www.ncc.go.jp/jp/information/press_release_20150428 html.

2. Japan Cancer Society, http://www.jcancer.jp/.

3. Formica V, Roselli M (2015) Targeted therapy in first line treatment of RAS wild type colorectal cancer. World J Gastroenterol 21: 2871-2874. [Crossref]

4. Peters GJ (2015) Therapeutic potential of TAS-102 in the treatment of gastrointestinal malignancies. Ther Adv Med Oncol 7: 340-356. [Crossref]

5. Lenz HJ, Stintzing S, Loupakis F (2015) TAS-102, a novel antitumor agent: a review of the mechanism of action. Cancer Treat Rev 41: 777-783. [Crossref]

6. Utsugi T (2013) New challenges and inspired answers for anticancer drug discovery and development. Jpn J Clin Oncol 43: 945-953. [Crossref]

7. Doi T, Ohtsu A, Yoshino T, Boku N, Onozawa Y, et al. (2012) Phase I study of TAS102 treatment in Japanese patients with advanced solid tumours. Br J Cancer 107: 429-434. [Crossref]

8. Grothey A, Van Cutsem E, Sobrero A, Siena S, Falcone A, et al. (2013) Regorafenib monotherapy for previously treated metastatic colorectal cancer (CORRECT): an international, multicentre, randomised, placebo-controlled, phase 3 trial. Lancet 381: 303-312. [Crossref]

9. Yoshino T, Mizunuma N, Yamazaki K, Nishina T, Komatsu Y, et al. (2012) TAS-102 monotherapy for pretreated metastatic colorectal cancer: a double-blind, randomised, placebo-controlled phase 2 trial. Lancet Oncol 13: 993-1001. [Crossref]

10. Mayer RJ, Van Cutsem E, Falcone A, Yoshino T, Garcia-Carbonero R, et al. (2015) Randomized trial of TAS-102 for refractory metastatic colorectal cancer. $N$ Engl J Med 372: 1909-1919. [Crossref]

11. Bendell JC, Rosen LS, Mayer RJ, Goldman JW, Infante JR, et al. (2015) Phase 1 study of oral TAS-102 in patients with refractory metastatic colorectal cancer. Cancer Chemother Pharmacol 76: 925-932. [Crossref]

12. Therasse P, Arbuck SG, Eisenhauer EA, Wanders J, Kaplan RS, et al., New guidelines to evaluate the response to treatment in solid tumors. European Organization for Research and Treatment of Cancer, National Cancer Institute of the United States, National Cancer Institute of Canada. J Natl Cancer Inst 92: 205-216. [Crossref]

13. Japanese Society for Cancer of the Colon and Rectum, http://www.jsccr.jp/guideline/ index_news.html\#g1601.

14. National Comprehensive Cancer Network, https://www.ncen.org/.

15. Heinemann V, von Weikersthal LF, Decker T, Kiani A, Vehling-Kaiser U, et al. (2014) FOLFIRI plus cetuximab versus FOLFIRI plus bevacizumab as first-line treatment for patients with metastatic colorectal cancer (FIRE-3): a randomised, open-label, phase 3 trial. Lancet Oncol 15: 1065-1075. [Crossref] 
Matsuda K (2017) Alteration of tumor markers may predict survival in colorectal cancer patients treated with TAS-102 or regorafenib as salvage-line chemotherapy: a single-institutional experience

16. Huang SC, Lin JK, Lin TC, Chen WS, Yang SH, et al. (2015) Concordance of Carcinoembryonic Antigen Ratio and Response Evaluation Criteria in Solid Tumors as Prognostic Surrogate Indicators of Metastatic Colorectal Cancer Patients Treated with Chemotherapy. Ann Surg Oncol 22: 2262-2268. [Crossref]
17. Tabernero J, Lenz HJ, Siena S, Sobrero A, Falcone A, et al. (2015) Analysis of circulating DNA and protein biomarkers to predict the clinical activity of regorafenib and assess prognosis in patients with metastatic colorectal cancer: a retrospective, exploratory analysis of the CORRECT trial. Lancet Oncol 16: 937-948. [Crossref]

Copyright: (C2017 Matsuda K. This is an open-access article distributed under the terms of the Creative Commons Attribution License, which permits unrestricted use, distribution, and reproduction in any medium, provided the original author and source are credited. 